\title{
QUALIDADE DA MADEIRA SERRADA PROVENIENTE DE ÁRVORES DOMINANTES E MÉDIAS DE Eucalyptus grandis SUBMETIDAS À SECAGEM
}

\author{
Joel Telles de Souza', Rômulo Trevisan², Luciano Denardi², Diego Martins Stangerlin³, Magnos Alan Vivian', \\ Clovis Roberto Haselein ${ }^{4}$, Elio José Santini ${ }^{5}$
}

(recebido: 16 de outubro de 2009; aceito: 25 de novembro de 2011)

\begin{abstract}
RESUMO: Neste estudo, objetivou-se verificar, em árvores de diâmetro dominante e médio, a influência da secagem ao ar livre e secagem convencional na qualidade da madeira serrada de um povoamento de Eucalyptus grandis W. Hill ex Maiden, aos 14 anos de idade, situado no município de Capivari do Sul, Rio Grande do Sul. Para tanto, a amostragem das árvores foi realizada após um inventário piloto, tendo como base o diâmetro à altura do peito (DAP) dominante e médio. Após a derrubada, foi retirada a tora localizada entre as posições DAP e $25 \%$ da altura comercial. As toras foram desdobradas em tábuas para serem submetidas à secagem ao ar livre até $18 \%$ do teor de umidade, e à secagem convencional foi conduzida até 13\% de umidade. Rachaduras de topo e arqueamento das tábuas foram avaliadas após o desdobro, na secagem ao ar livre e na secagem convencional. Os resultados mostraram que, tanto para as árvores dominantes quanto para as médias, o índice de rachaduras de topo e a flecha de arqueamento das tábuas não diferem significativamente nas diferentes fases de processamento da madeira. No entanto, a comparação entre esses dois estratos evidencia que a magnitude desse defeito é mais pronunciada nas árvores médias.
\end{abstract}

Palavras-chave: Rachaduras de topo, arqueamento, secagem ao ar livre, secagem convencional, madeira refratária.

\section{WOOD QUALITY OF Eucalyptus grandis DOMINANT AND AVERAGE TREES SUBMITTED TO DRYING}

\begin{abstract}
This study determined, in trees of dominant and average diameter, the influence of air seasoning followed by kiln drying in the sawn wood quality of 14 years old stand of Eucalyptus grandis W. Hill ex Maiden, located in Capivari do Sul municipality, state of Rio Grande do Sul. The sampling of trees was performed after a pilot inventory on the basis of the dominant and average diameter at breast height (DBH). After cutting down the trees, there were selected the logs located between the DBH and $25 \%$ of commercial height. The logs were then sawn using into boards that were submitted to air seasoning from green up to $18 \%$ of moisture content, followed by kiln drying until 13\% of moisture content. End checking and board crook were evaluate after sawing, following air seasoning and kiln drying. The results indicate that, both for dominant and average trees, end checking and board crook were not influenced by different wood process stage. However, the contrast between dominant and average trees shows that the magnitude of defects is higher in the average trees.
\end{abstract}

Key words: End checking, crook, air seasoning, kiln drying, refractory wood.

\section{INTRODUÇÃO}

Atualmente, diante da pressão ambiental exercida acerca da exploração de florestas nativas como fonte de matéria-prima, os reflorestamentos tornaram-se importantes alternativas na confecção de produtos madeira. Entretanto, com um mercado em crescimento e cada vez mais comprometido com qualidade, seria fora de propósito abster-se da derrubada dessas matas nativas se não houvesse uma opção adequada tanto no aspecto ecológico quanto no produtivo. Assim, aliado à elevação do valor econômico e escassez das madeiras nobres, o uso diversificado do gênero Eucalyptus vem apresentando um papel fundamental no setor florestal brasileiro.

Segundo pesquisa feita pelo Instituto Nacional de Pesquisas Espaciais - INPE (2010), a área de desmatamento

\footnotetext{
${ }^{1}$ Engenheiro Florestal, Mestrando em Engenharia Florestal-Programa de Pós-Graduação em Engenharia Florestal-Centro de Ciências Rurais-Universidade Federal de Santa Maria - Av. Roraima, 1000, Bairro Camobi - 97105-900 - Santa Maria, RS - joeltelles@hotmail.com, magnosalan@yahoo.com.br ${ }^{2}$ Engenheiro Florestal, Professor Dr. em Engenharia Florestal - Departamento de Ciências Florestais - Centro de Educação Superior Norte Universidade Federal de Santa Maria - BR386, km 40 - Linha 7 de Setembro - 98400-000 - Frederico Westphalen, RS - trevisanr@smail.ufsm.br, lucianodenardi@yahoo.com.br

${ }^{3}$ Engenheiro Florestal, Doutorando em Ciências Florestais - Departamento de Engenharia Florestal - Universidade de Brasília - SQS 406, Bloco A, ap. 306 - Asa Sul - 70255-010 - Brasília, DF - diego_stangerlin@yahoo.com.br

${ }^{4}$ Engenheiro Florestal, Professor Dr. em Forest Products - Departamento de Ciências Florestais - Centro de Ciências Rurais - Universidade Federal de Santa Maria - Av. Roraima 1000, Bairro Camobi - 97105-900 - Santa Maria, RS - clovis.haselein@smail.ufsm.br

${ }^{5}$ Engenheiro Florestal, Professor Dr. em Engenharia Florestal - Departamento de Ciências Florestais - Centro de Ciências Rurais - Universidade Federal de Santa Maria - Av. Roraima 1000, Bairro Camobi - 97105-900 - Santa Maria, RS - eliosantini@smail.ufsm.br
}

Cerne, Lavras, v. 18, n. 1, p. 167-174, jan./mar. 2012 
na Amazônia Legal caiu mais de 4 vezes do ano de 2004 para 2010, enquanto que o plantio de eucalipto vem numa crescente.

Outro fator importante que favorece a utilização de florestas plantadas é a tendência mundial de expansão dos mercados para a "madeira ambientalmente correta", ou seja, certificada com o chamado "selo verde". Aliando todos esses fatores com a globalização dos mercados consumidores, tem-se uma crescente necessidade de aumento na produtividade e de atendimento de padrões de qualidade cada vez mais exigentes, estimulando a exploração da madeira de espécies exóticas como, por exemplo, as pertencentes ao gênero Eucalyptus (TREVISAN, 2010).

De acordo Marques (2008), o conhecimento da variabilidade dessa madeira quando exposta às condições de secagem, por exemplo, pode resultar em benefícios para o controle de possíveis defeitos e aumentar a qualidade dos produtos. Da mesma forma Jankowsky (1995) revela que a adequada secagem da madeira serrada, antes da sua transformação em bens e produtos, é reconhecidamente a fase mais importante de todo o processamento que visa a agregar valor ao produto final.

Assim, considerando os aspectos econômicos e de qualidade da madeira do gênero Eucalyptus, autores como Andrade (2000), Calonego (2004), Ciniglio (1998), Jankowsky (2008), Martins et al. (1999), Northway (1996), Pratt (1974), Rozas (1993) e Santos (2002), recomendam o uso de um potencial de secagem ameno e de temperaturas iniciais que não excedam a $45^{\circ} \mathrm{C}$, principalmente nos estágios iniciais. Outra alternativa para atender a esses aspectos caracteriza-se pelo uso da pré-secagem ou a combinação da secagem ao ar livre com posterior secagem convencional, proporcionando redução de custos e otimização do processo, por meio da maior eficiência das câmaras convencionais de secagem; além de atenuar o gradiente de umidade na madeira, responsável pela maioria dos defeitos durante a secagem (CAMPBELL; HARTLEY, 1988; CINIGLIO, 1998; DENIG et al., 2000; FRANZONI, 2001; JANKOWSKY, 2008; NORTHWAY, 1996; SANTOS, 2002; STÖHR, 1977).

Para Jankowsky (1995) e Martins (1988), os defeitos de secagem e as rachaduras associadas às tensões de crescimento trazem como resultado perdas significativas de madeira durante o processamento industrial, sendo considerado como um dos principais entraves à utilização econômica de espécies de eucalipto.

Cerne, Lavras, v. 18, n. 1, p. 167-174, jan./mar. 2012
De acordo com Ferreira et al. (2005) e Rocha e Tomaselli (2002) existem várias técnicas que podem ser adotadas, tanto na obtenção e preparo das toras, como no desdobro para reduzir as manifestações das tensões de crescimento na obtenção de madeira serrada. Segundo Néri et al. (2006) e Vidaurre et al. (2007), o método e a força que se exerce no corte de uma peça, influenciam diretamente na qualidade da mesma, já que os variados tipos de cortes envolvem um processo de tensão e ruptura na madeira. Estudos indicam que é possível minimizar os efeitos das tensões internas, mas não eliminá-los (HILLS; BROWN, 1978; JARA et al., 1997; LUZ et al., 1992; OLIVEIRA, 1999). Dentre os defeitos mais frequentes causados pelas tensões internas na madeira serrada de eucalipto, encontram-se rachaduras de topo, rachaduras superficiais, arqueamentos, encanoamentos e encurvamentos (VERMAAS, 2000).

Conforme Galvão e Jankowsky (1985) e Jankowsky (2005), os empenamentos são caracterizados por serem distorções que ocorrem na madeira em relação aos planos originais de sua superfície. Galvão e Jankowsky (1985), Gomide (1973), Jankowsky (2005) e Ponce Herrero e Watai (1985), definem o empenamento longitudinal como sendo uma curvatura da peça no eixo em questão (sentido do comprimento) e em relação à superfície de maior dimensão. De maneira geral, originam-se das diferenças inerentes às contrações radial, tangencial e longitudinal, porém, irregularidades na grã, presença de lenho de reação e lenho juvenil, também influenciam o aparecimento de peças empenadas ao final da secagem (JANKOWSKY, 2005; SANTINI, 1992).

A manifestação de peças arqueadas ao final da secagem é um dos defeitos mais problemáticos com relação ao seu controle, uma vez que, durante a secagem, as tábuas ficam dispostas sem qualquer restrição lateral que as impeça de trabalharem. Outro defeito de grande relevância quanto ao seu controle são as rachaduras de topo, que por vezes limitam a utilização da madeira serrada, em especial as peças de eucalipto, visto que a espécie é bastante susceptível em razão da presença das tensões de crescimento.

Visando a reduzir os defeitos e, consequentemente, aumentar o aproveitamento de madeira processada, esse trabalho foi desenvolvido com o objetivo de verificar em madeira serrada, proveniente de árvores de diâmetro dominante e médio, o efeito da secagem ao ar livre e da secagem convencional, na qualidade da madeira de Eucalyptus grandis W. Hill ex Maiden, aos 14 anos de idade. 


\section{MATERIAL E MÉTODO}

\subsection{Características do experimento}

A área experimental localiza-se nas coordenadas geográficas de $50^{\circ} 30^{\prime}$ de longitude Oeste e $30^{\circ} 08^{\prime}$ de latitude Sul, no município de Capivari do Sul, litoral Norte do Estado do Rio Grande do Sul.

Os dados para o presente estudo foram obtidos de um povoamento de Eucalyptus grandis instalado em maio de 1990 e implantado com espaçamento inicial de 3,0 x 1,7 m. A amostragem das árvores foi realizada em 2004, portanto aos 14 anos de idade, com base no diâmetro à altura do peito (DAP). Foram coletadas 16 árvores dominantes, com diâmetro médio de $37,1 \pm 2,1 \mathrm{~cm}$, e 16 árvores médias, com diâmetro médio de 27,0 $\pm 5,5 \mathrm{~cm}$, totalizando 32 árvores.

\subsection{Determinação dos defeitos da madeira serrada}

Para a determinação da qualidade da madeira serrada foi selecionada a primeira tora da árvore dominante e média amostrada, localizada entre a posição do DAP e $25 \%$ da altura comercial.

Essas foram transportadas até a serraria da empresa, onde com auxílio da serra de fita dupla, cortaram-se as costaneiras, e posteriormente, utilizando a serra circular múltipla, transformaram-se os blocos em tábuas, com dimensões comerciais médias de 2,5 x 15,5 x 330,0 cm, totalizando 122 amostras, ou seja, 61 tábuas para cada estrato estudado (Figura 1).

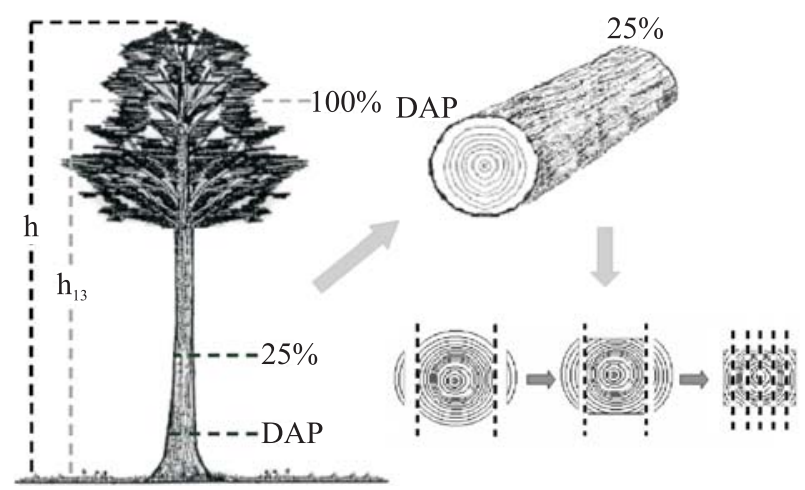

Figura 1 - Esquema ilustrativo da seleção da tora e confecção das tábuas.

Figure 1 - Schematic illustration of the selection of logs and confection of the board.

A primeira avaliação da qualidade desse material em relação a rachaduras de topo e arqueamento foi realizada após o desdobro, sendo denominada como Condição Pós-desdobro. As tábuas foram empilhadas de forma horizontal para secagem ao ar livre, onde permaneceram até massa constante, para nova análise (Condição Pós-secagem ao ar livre). Para constatar a massa constante, selecionaram-se algumas tábuas, que foram pesadas periodicamente. Essa etapa teve duração de, aproximadamente, seis meses e o teor de umidade médio de equilíbrio foi de aproximadamente 18\%. Após esse período, realizou-se a secagem do material em estufa convencional até um teor de umidade final igual a 13\%, sendo então efetuada a última avaliação (Condição Póssecagem em estufa).

\subsection{Rachaduras de topo das tábuas}

O índice de rachaduras de topo das tábuas foi determinado pelo somatório dos seus comprimentos individuais, em cada lado. $\mathrm{O}$ valor percentual do índice de rachaduras foi obtido pela razão entre a média dos seus comprimentos e o comprimento total da tábua, conforme Equação 1 (INSTITUTO BRASILEIRO DE DESENVOLVIMENTO FLORESTAL - IBDF, 1983).

$$
\mathrm{IR}_{\mathrm{tb}}=\left[\frac{\sum \mathrm{LR}_{\mathrm{R}}}{2 \mathrm{Lr}_{\mathrm{r}}}\right] 100
$$

em que: $\mathrm{IR}_{\mathrm{tb}}=$ índice de rachadura de topo da tábua, em $\% ; \sum \mathrm{L}_{\mathrm{R}}=$ somatório dos comprimentos das rachaduras de topo nas duas faces da tábua, em $\mathrm{cm} ; \mathrm{Lr}=$ comprimento total da tábua, em $\mathrm{cm}$.

\subsection{Arqueamento das tábuas}

Entende-se por arqueamento a curvatura ao longo do comprimento da peça de madeira num plano paralelo à face, sendo determinado pela Equação 2 (IBDF, 1983).

$$
\mathrm{AR}_{\mathrm{tb}}=\left[\frac{\mathrm{X}}{\mathrm{Lr}}\right] 100
$$

em que: $\mathrm{AR}_{\mathrm{tb}}=$ arqueamento da tábua, em \%; $\mathrm{X}$ = flecha máxima, em cm; Lr = comprimento total da tábua, em cm.

\subsection{Análise estatística dos dados}

Os dados médios das rachaduras de topo e do arqueamento das tábuas confeccionadas a partir das árvores dominantes e médias em função da condição de secagem da madeira foram submetidos à análise de variância e, no caso de rejeição da hipótese de igualdade de médias, ao teste de médias (Least Significant Difference LSD, $\alpha=5 \%$ ).

Cerne, Lavras, v. 18, n. 1, p. 167-174, jan./mar. 2012 


\section{RESULTADOS E DISCUSSÃO}

\subsection{Rachaduras de topo das tábuas}

Os resultados encontrados para o índice de rachaduras de topo das tábuas, tanto para as árvores dominantes (Prob. $>\mathrm{F}=0,0654$ ) quanto para as médias (Prob. $>\mathrm{F}=0,6384$ ), aos 14 anos de idade, evidenciam que não houve influência significativa da condição de processamento em nível de 5\% de probabilidade de erro.

Analisando as médias de rachaduras de topo das tábuas em função da secagem da madeira na Figura 2, é interessante observar que, apesar das diferenças entre os índices de rachaduras para as três condições de secagem não serem significativas, houve um acréscimo de, aproximadamente, 3,0\% nesses índices, tanto para as árvores dominantes quanto para as médias.

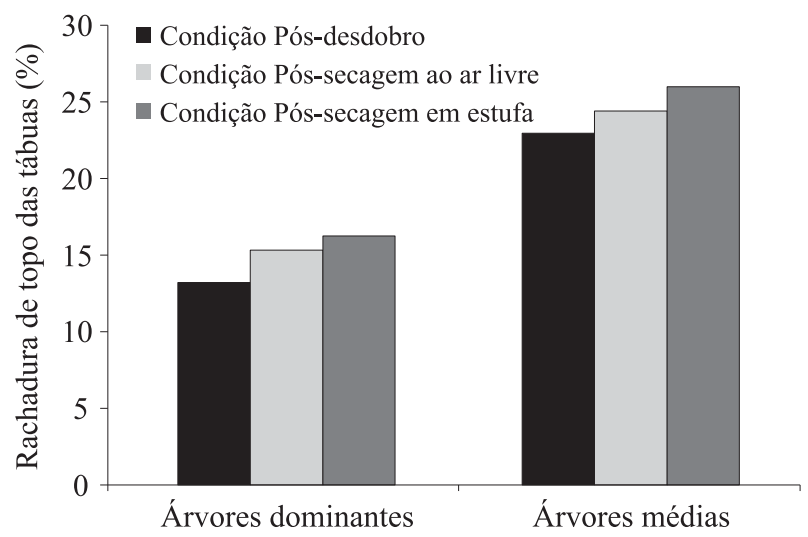

Figura 2 - Médias das rachaduras de topo das tábuas em função da secagem da madeira para árvores dominantes e médias.

Figure 2 - End checking average in function of the drying wood of the dominant and average trees.

Rocha e Tomaselli (2002), avaliando a qualidade da madeira serrada de Eucalyptus grandis e Eucalyptus dunnii, também evidenciaram valores não significativos entre os índices de rachaduras de topo na condição verde e após a secagem, com valores médios de rachaduras para o Eucalyptus grandis muito semelhantes ao do presente estudo, $14,65 \%$ e $23,51 \%$, respectivamente, para toras mais grossas e toras mais finas. Entretanto, Rocha e Trugilho (2006), estudando a espécie Eucalyptus dunnii, relatam um aumento significativo das rachaduras de topo em função dessas condições de processamento.

Comparando esse índice entre as árvores dominantes e médias verifica-se que existe diferença significativa em nível $\alpha=5 \%$ de probabilidade de erro e Prob. $>\mathrm{F}=0,0001$. Na Tabela 1, é apresentado o teste de médias das rachaduras de topo das tábuas em relação aos estratos amostrados.

Tabela 1 - Comparação de médias do parâmetro rachadura de topo das tábuas das árvores dominantes e médias.

Table 1 - Comparison of average of the parameter end checking of the dominant and average trees.

\begin{tabular}{lc}
\hline Estrato amostrado $^{1}$ & $\mathrm{IR}_{\mathrm{tb}}(\%)^{2}$ \\
\hline Dominante & $15,1 \mathrm{a}^{3}$ \\
Médio & $23,7 \mathrm{~b}$ \\
\hline
\end{tabular}

${ }^{1}$ Árvores de diâmetro dominante e médio.

${ }^{2} \mathrm{IR}_{\mathrm{tb}}=$ médias dos índices de rachaduras de topo das tábuas das árvores dominantes e médias, em \%.

${ }^{3}$ Médias seguidas pela mesma letra em cada coluna não diferem significativamente entre si em nível de $5 \%$ de probabilidade de erro; teste de médias (LSD = Least Significant Difference).

Lopes et al. (2004), estudando agrupamento de árvores matrizes de Eucalyptus grandis com diferentes diâmetros, também observou que o estrato dominante apresentou menor valor percentual de rachaduras de topo na madeira serrada, em comparação às árvores de menor diâmetro. Resultados semelhantes foram encontrados por Chafe (1981) e Fernandes e Ferreira (1986), os quais concluem que a intensidade dessas rachaduras diminui com o aumento do diâmetro das toras.

Entretanto, para Calonego et al. (2006), Calonego e Severo (2005), Chafe (1985), Malan (1984), Rozas (1993) e Wilson (1985), essa variação entre árvores dominantes e médias apresentou uma tendência contrária, ou seja, a intensidade das rachaduras aumentou, proporcionalmente, com o diâmetro das toras. Wilson (1985) presumiu que isso pode ocorrer, em decorrência da maior proporção de madeira juvenil do que adulta.

De outra forma, resultados encontrados por Lima (2005) e Lima et al. (2007) relatam que o índice de rachaduras de extremidade de tora não foi influenciado pela classe de diâmetro. Santos (2002) afirma que não existe uma metodologia padrão para quantificação das rachaduras das tábuas e que cada autor adota seu próprio índice de quantificação desses defeitos, determinando a sua própria amostragem para quantificar essas rachaduras. Segundo o mesmo autor, tal fato apresenta-se como um entrave para a comparação entre os trabalhos descritos na literatura.

Cerne, Lavras, v. 18, n. 1, p. 167-174, jan./mar. 2012 


\subsection{Arqueamento}

O resumo da análise de variância para arqueamento das tábuas evidencia que tanto as árvores dominantes (Prob. $>\mathrm{F}=0,8022$ ) quanto às médias (Prob. $>\mathrm{F}=$ 0,9980), aos 14 anos de idade, não foram influenciadas significativamente pela condição de processamento, em nível de 5\% de probabilidade de erro. Na Figura 3, apresentam-se as médias desse defeito nas tábuas, em função das diferentes condições de processamento para os dois estratos estudados.

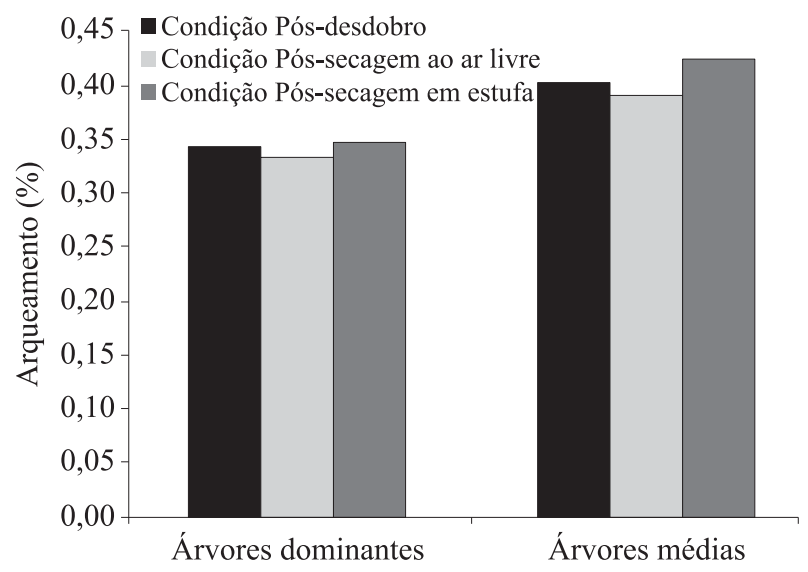

Figura 3 - Médias de arqueamento das tábuas em função da condição de processamento da madeira para árvores dominantes e médias.

Figure 3 - Board crook average in function of the wood processing condition of the dominant and average trees.

Na Figura 3, verifica-se que, tanto para as árvores dominantes quanto para as médias, todos os valores de arqueamento das tábuas em função da condição de processamento estão de acordo com a Norma para Classificação de Madeira Serrada de Folhosas (IBDF, 1983), que regulamenta um máximo de defeito igual a 0,5\% em relação ao comprimento total da tábua.

Resultado semelhante ao que foi encontrado por Rocha e Tomaselli (2002) e Rocha e Trugilho (2006) que, estudando a qualidade da madeira de Eucalyptus dunnii e Eucalyptus grandis, não encontraram diferença significativa nos valores de arqueamento das tábuas, nas condições de processamento da madeira verde e após sua secagem.

Comparando o arqueamento das tábuas entre as árvores dominantes e médias, verifica-se que existe diferença significativa em nível de 5\% de probabilidade de erro e Prob. $>F=0,019$. Na Tabela 2, é apresentado o teste de comparação de médias do arqueamento das tábuas em relação aos estratos amostrados.

Tabela 2 - Comparação de médias do parâmetro arqueamento das tábuas das árvores dominantes e médias.

Table 2 - Comparison of average of the parameter board crook of the dominant and average trees.

\begin{tabular}{lc}
\hline Estrato amostrado $^{1}$ & $\mathrm{AR}_{\mathrm{tb}}(\%)^{2}$ \\
\hline Dominante & $0,34 \mathrm{a}^{3}$ \\
Médio & $0,41 \mathrm{~b}$ \\
\hline
\end{tabular}

${ }^{1}$ Árvores de diâmetro dominante e médio.

${ }^{2} \mathrm{AR}_{\mathrm{tb}}=$ médias do arqueamento das tábuas das árvores dominantes e médias, em \%.

${ }^{3}$ Médias seguidas pela mesma letra em cada coluna não diferem significativamente entre si em nível de 5\% de probabilidade de erro; teste de médias (LSD = Least Significant Difference).

Calonego et al. (2006), estudando a espécie Eucalyptus grandis relatam que os maiores índices desse defeito foram encontrados nas tábuas das árvores de maiores diâmetros, com $0,42 \%$, enquanto que as de menores diâmetros tiveram $0,36 \%$ de arqueamento. Entretanto, Lima (2005) e Rocha e Tomaselli (2002) analisando esse defeito em Eucalyptus grandis e Eucalyptus dunnii, citam que não ocorreu diferença significativa nas diferentes classes diamétricas, nas mesmas condições de umidade da madeira.

Segundo Lima (2005), essas diferenças entre resultados podem ser explicadas pelo deslocamento da medula ou excentricidade natural da tora. A presença dessa característica provoca um encurvamento do semibloco, durante o desdobro, mesmo quando efetuado numa serra de corte simétricos em relação à medula da tora, como foi o caso da serra fita dupla utilizada no presente estudo. $\mathrm{O}$ encurvamento do semibloco transforma-se, naturalmente, em arqueamento das tábuas obtidas desse mesmo semibloco durante o corte na serra múltipla subsequente. Na atual pesquisa, não foi analisada essa característica, mas a diferença de arqueamento constatada entre as árvores médias e dominantes pode ter ocorrido pela presença de deslocamento da medula.

Embora exista forte controle genético na expressão da característica rachadura de topo e arqueamento de tábuas, o aprimoramento das tecnologias de abate, processamento e secagem de eucalipto, pode contribuir de maneira significativa na viabilização da madeira desse gênero para uso na indústria madeireira.

Cerne, Lavras, v. 18, n. 1, p. 167-174, jan./mar. 2012 


\section{CONCLUSÕES}

Em função dos resultados obtidos conclui-se que:

O índice de rachadura de topo e arqueamento das tábuas não tiveram influência significativa nas diferentes condições de Pós-desdobro, Pós-secagem ao ar livre e Pós-secagem em estufa, tanto para as árvores dominantes quanto para as médias.

Embora não significativa essa diferença entre os índices de rachadura de topo das tábuas para as três condições de processamento, houve um acréscimo constante de, aproximadamente, 3,0\% entre esses índices, para árvores dominantes e médias. O mesmo não ocorreu com o arqueamento.

Comparando os defeitos de rachadura de topo e arqueamento das tábuas entre árvores dominantes e médias, verificou-se diferença significativa entre esses estratos.

No entanto, a comparação entre os dois estratos evidencia que, a magnitude desses defeitos é mais pronunciada nas árvores médias. Sendo as dominantes, portanto, classificadas como superiores no processo de secagem.

\section{REFERÊNCIAS}

ANDRADE, A. Indicação de programas para secagem convencional de madeiras. 2000. 72 p. Dissertação (Mestrado) - Escola Superior de Agricultura "Luiz de Queiroz”, Piracicaba, 2000.

CALONEGO, F. W. Estimativa do tempo de vaporização das toras e sua implicação no desdobro e na secagem de Eucalyptus grandis. 2004. 120 f. Dissertação (Mestrado em Energia na Agricultura) - Universidade Estadual Paulista, Botucatu, 2004.

CALONEGO, F. W.; SEVERO, E. T. D. Efeito do diâmetro de toras na magnitude das tensões de crescimento de Eucalyptus grandis. Energia na Agricultura, Botucatu, v. 20, n. 2, p. 53-65, 2005.

CALONEGO, F. W.; SEVERO, E. T. D.; CONEGLIAN, A.; BARREIROS, R. M. Qualidade da secagem de Eucalyptus grandis mediante vaporização simultânea em toros e em madeira serrada. Silva Lusitana, Lisboa, v. 14, n. 2, p. 169-180, 2006.

CAMPBELL, G. S.; HARTLEY, J. Drying and dried wood. In: HILLIS, W. E.; BROWN, A. G. (Ed.). Eucalyptus for

Cerne, Lavras, v. 18, n. 1, p. 167-174, jan./mar. 2012 wood production. Melbourne: Academic, 1988. p. 328336.

CHAFE, S. C. Variations in longitudinal growth stress, basic density and modulus of elasticity with height in tree. Australian Forest Research, Melbourne, v. 11, n. 1, p. 79-82, 1981.

CHAFE, S. C. Variations in longitudinal with height in trees of Eucalyptus nitens Maiden. Australian Forest Research, Melbourne, v. 15, n. 1, p. 51-55, 1985.

CINIGLIO, G. Avaliação da secagem de madeira serrada de Eucalyptus grandis e Eucalyptus urophylla. 1998. 69 f. Dissertação (Mestrado) - Escola Superior de Agricultura "Luiz de Queiroz”, Piracicaba, 1998.

DENIG, J.; WENGERT, E. M.; SIMPSON, W. T. Drying hardwood lumber. Madison: USDA, 2000. 138 p.

FERNANDES, P. S.; FERREIRA, M. C. Tensões de crescimento em toras de Eucalyptus saligna e suas relações com os diâmetros. São Paulo: Instituto Florestal, 1986. 17 p.

FERREIRA, S.; LIMA, J. T.; ROSADO, S. C. da S.; TRUGILHO, P. F. Influência do desdobro no rendimento e qualidade do eucalipto. Revista da Madeira, Curitiba, n. 92, p. 15-22, out. 2005.

FRANZONI, J. A. Utilização da madeira de eucalipto na indústria de móveis e padrões de qualidade de madeira para móveis de exportação. In: SEMINÁRIO MADEIRA DE EUCALIPTO: TENDÊNCIAS E USOS, 2001, Curitiba. Anais... Curitiba: FUPEF, 2001. p. 50-52.

GALVÃO, A. P. M.; JANKOWSKY, I. P. Secagem racional da madeira. São Paulo: Nobel, 1985. 111 p.

GOMIDE, J. L. Secagem da madeira. Viçosa, MG: UFV, 1973. $76 \mathrm{p}$.

HILLIS, W. E.; BROWN, A. G. (Ed.). Eucalypts of wood production. Melbourne: CSIRO, 1978. 434 p.

INSTITUTO BRASILEIRO DE DESENVOLVIMENTO FLORESTAL. Norma para classificação de madeira serrada de folhosas. Brasília: Ministério da Agricultura, 1983. $67 \mathrm{p}$. 
INSTITUTO NACIONAL DE PESQUISAS ESPACIAIS.

Pesquisa. São José dos Campos, 2010.

JANKOWSKY, I. P. Equipamentos e processos para secagem de madeiras. Revista da Madeira, Curitiba, n. 115, p. 43-48, jul. 2008.

JANKOWSKY, I. P. Equipamentos e processos para a secagem de madeiras. In: SEMINÁRIO INTERNACIONAL DE UTILIZAÇÃO DA MADEIRA DE EUCALIPTO PARA SERRARIA, 1995, São Paulo. Anais... São Paulo: IPEF/IPT/IUFRO/LCF/ESALQ/USP, 1995. p. 109-118.

JANKOWSKY, I. P. Secagem adequada é decisiva para qualidade. Revista da Madeira, Curitiba, n. 89, p. 33-37, abr. 2005.

JARA, E. R. P.; MIRANDA, M. J. de A. C.; HUPHREYS, R. D. Influência do tratamento térmico na redução das tensões internas de crescimento em toras de Eucalyptus grandis. In: IUFRO CONFERENCE ON SILVICULTURE AND IMPROVEMENT OF EUCALYPTS, 1997, Salvador. Proceedings... Colombos: EMBRAPA, 1997. p. 276-280.

LIMA, I. L. Influência do desbaste e da adubação na qualidade da madeira serrada de Eucalyptus grandis Hill ex Maiden. 2005. 137 f. Tese (Doutorado em Recursos Florestais) - Escola Superior de Agricultura "Luiz de Queiroz”, Piracicaba, 2005.

LIMA, I. L.; GARCIA, J. N.; STAPE, J. L. Influência do desbaste e da fertilização no deslocamento da medula e rachaduras de extremidade de tora de Eucalyptus grandis Hill ex-Maiden. Cerne, Lavras, v. 13, n. 2, p. 170-177, abr./jun. 2007.

LOPES, M. de C.; HASELEIN, C. R.; SANTINI, E. J.; LONGHI, S. J.; SILVIANA, R.; FERNANDES, D. L. G.; MENEZES, L. F. Agrupamento de árvores matrizes de Eucalyptus grandis em função das variáveis dendrométricas e das características tecnológicas da madeira. Ciência Florestal, Santa Maria, v. 14, n. 2, p. 133-144, 2004.

LUZ, H. F.; MELZER, J. F.; COUTINHO, C. J.; FERREIRA, M. C. Manejo de florestas de eucalipto para usos múltiplos. Piracicaba: Eucatex Florestal, 1992. 30 p.
MALAN, F .S. Studies on the phenotypic variation in growth stress intensity and its association with tree and wood properties of South African Grow Eucalyptus grandis (Hill ex-Maiden). 1984. 272 f. Thesis (Ph.D.) - University of Stellenbosch, Stellenbosch, 1984.

MARQUES, M. H. B. Agrupamento de 41 espécies de madeiras da Amazônia para secagem baseado em características anatômicas e físicas. 2008. 125 f. Tese (Doutorado em Ciencias Florestais) - Universidade de Brasilia, Brasilia, 2008.

MARTINS, V. A. Secagem de madeira serrada. Brasilia: IBDF, 1988. 56 p.

MARTINS, V. A.; GOUVEIA, F. N.; MARTINEZ, S. Secagem convencional de madeira de eucalipto. In: CONGRESSO INTERNACIONAL SOBRE ECOSSISTEMAS FLORESTAIS, 5., 1999, Curitiba. Anais... Curitiba: Biosfera, 1999. Poster.

NÉRI, A. C.; GONÇALVES, R.; HERNANDES, R. E. Estilo do corte influi na qualidade da madeira. Revista da Madeira, Curitiba, n. 98, p. 12-15, ago. 2006.

NORTHWAY, R. L. Drying strategies for plantation-grown eucalypts. In: INTERNATIONAL IUFRO WOOD DRYING CONFERENCE, 5., 1996, Quebec. Proceedings... Quebec: IUFRO, 1996. p. 289-296.

OLIVEIRA, J. T. S. Problemas e oportunidades com a utilização da madeira de Eucalipto. In: WORKSHOP: TÉCNICAS DE ABATE, PROCESSAMENTO E UTILIZAÇÃO DA MADEIRA DE EUCALIPTO, 1999, Viçosa, MG. Anais... Viçosa, MG: UFV, 1999. p. 39-52.

PONCE HERRERO, R.; WATAI, L. T. Manual de secagem da madeira. Brasília: MIC/STI/IPT, 1985. 70 p. (Série documentos, 22).

PRATT, G. H. Timber drying manual. London: Department of the Environment, Building Research Establishment, 1974. $152 \mathrm{p}$.

ROCHA, M. P.; TOMASELLI, I. Efeito do modelo de desdobro na qualidade da madeira serrada de Eucalyptus grandis e Eucalyptus dunnii. Cerne, Lavras, v. 8, n. 2, p. 7083, 2002.

Cerne, Lavras, v. 18, n. 1, p. 167-174, jan./mar. 2012 
ROCHA, M. P.; TRUGILHO, P. F. Qualidade de madeira serrada de Eucalyptus dunnii em função do método de desdobro e condição de umidade. Cerne, Lavras, v. 12, n. 4, p. 314-321, out./dez. 2006.

ROZAS, M. E. C. E. Contribuição ao desenvolvimento tecnológico para a utilização de madeira serrada de Eucalyptus grandis (Hill Ex Maiden) na geração de produtos com maior valor agregado. 1993. 133 p. Dissertação (Mestrado em Engenharia Florestal) Universidade Federal do Paraná, Curitiba, 1993.

SANTINI, E. J. Metodos de secagem de madeira. In: SEMINÁRIO SOBRE SECAGEM DE MADEIRA, 1., 1992, Santa Maria. Anais... Santa Maria: UFSM/CEPEF/FATEC, 1992. p. 47-59.

SANTOS, G. R. V. Otimização da secagem da madeira de Eucalyptus grandis (Hill ex Maiden). 2002. $70 \mathrm{f}$.

Dissertação (Mestrado em Ciências e Tecnologia da Madeira)

- Escola Superior de Agricultura “Luiz de Queiroz”,

Piracicaba, 2002.
STÖHR, H. P. The seasoning of South African grown Eucalyptus grandis and Eucalyptus saligna. South African Forestry Journal, Pretoria, v. 102, p. 61-66, 1977.

TREVISAN, R. Efeito do desbaste nos parâmetros dendrométricos e na qualidade da madeira de Eucalyptus grandis W. Hill ex Maiden. 2010. Tese (Doutorado em Engenharia Florestal) - Universidade Federal de Santa Maria, Santa Maria, 2010.

VERMAAS, H. F. A. Review of drying technology for young fast-grown eucalypts. In: THE FUTURE OF EUCALYPTUS FOR WOOD PRODUCTS, 2000, Launceston. Proceedings... Launceston: IUFRO, 2000. p. 193-203.

VIDAURRE, G.; VITAL, B. R.; SILVA, J. de C.; OLIVEIRA, J. T. S. Madeira serrada de eucalipto. Revista da Madeira, Curitiba, n. 102, p. 8-12, jan. 2007.

WILSON, P. J. Splitting studies on logs of Eucalyptus macarthurii, Eucalyptus elata, and Eucalyptus radiata. Pietermaritzburg: University of Natal, 1985.

Cerne, Lavras, v. 18, n. 1, p. 167-174, jan./mar. 2012 